\title{
ON THE BITS COUNTING FUNCTION OF REAL NUMBERS
}

\author{
TANGUY RIVOAL
}

\author{
(Received 27 June 2006; accepted 20 December 2006)
}

Communicated by W. W. L. Chen

\begin{abstract}
Let $B_{n}(x)$ denote the number of 1's occurring in the binary expansion of an irrational number $x>0$. A difficult problem is to provide nontrivial lower bounds for $B_{n}(x)$ for interesting numbers such as $\sqrt{2}, e$ or $\pi$ : their conjectural simple normality in base 2 is equivalent to $B_{n}(x) \sim n / 2$. In this article, amongst other things, we prove inequalities relating $B_{n}(x+y), B_{n}(x y)$ and $B_{n}(1 / x)$ to $B_{n}(x)$ and $B_{n}(y)$ for any irrational numbers $x, y>0$, which we prove to be sharp up to a multiplicative constant. As a by-product, we provide an answer to a question raised by Bailey et al. (D. H. Bailey, J. M. Borwein, R. E. Crandall and C. Pomerance, 'On the binary expansions of algebraic numbers', J. Theor. Nombres Bordeaux 16(3) (2004), 487-518) concerning the binary digits of the square of a series related to the Fibonacci sequence. We also obtain a slight refinement of the main theorem of the same article, which provides a nontrivial lower bound for $B_{n}(\alpha)$ for any real irrational algebraic number. We conclude the article with effective or conjectural lower bounds for $B_{n}(x)$ when $x$ is a transcendental number.
\end{abstract}

2000 Mathematics subject classification: primary 11K16; secondary 11J68, 68R01.

Keywords and phrases: binary expansions, algebraic numbers.

\section{Introduction}

The integer $B(m)$ is defined to be the number of 1's in the finite binary expansion of the nonnegative integer $m$, with $B(0)=0$. It is a classical fact that $B$ is sub-additive and sub-multiplicative, that is, that for all integers $m, n \geq 0$, the two inequalities $B(m+n) \leq B(m)+B(n)$ and $B(m n) \leq B(m) B(n)$ hold: see [5, 21] or Section 2, where we provide a proof for the sake of completeness. These inequalities are sharp, as the examples $10+1=11$ and $111 \times 100100=111111$ (in base 2) show. The behavior of $B\left(m^{j}\right)$ ( $m$ fixed and $j \rightarrow+\infty$ ) was studied in $[15,21]$.

In this article, we first prove related results when $m$ is allowed to be an irrational number (in Theorem 1) via a study of the function

$$
B_{n}(x)=\#\left\{j \leq n \mid x_{j}=1\right\}
$$

(c) 2008 Australian Mathematical Society 1446-7887/08 \$A2.00+0.00 
where $n \geq 0$ and $x=\left(x_{-p} \ldots x_{-1} x_{0}, x_{1} x_{2} x_{3} \ldots\right)_{2}$ is the binary expansion of $x \geq 0$. The case where $x$ is a rational number of the form $n / 2^{m}(n, m \in \mathbb{N})$ is ambiguous: in this case, there are two possible binary expansions, finite and infinite. The only rational numbers of this form that we will consider in this text are the integers and we only use their finite binary expansion; with this convention, for any positive integer $x$ (written in its finite binary expansion), we have $B_{n}(x)=B_{0}(x)=B(x)$. In any occurrence of the quantity $B_{n}(x)$, it will be tacitly supposed (if not explicitly stated) that $x$ is either an irrational number or an integer.

Theorem 1 has some interesting consequences, which we now describe as a motivation for the article. We recall that a real number $x$ is said to be simply normal in base 2 if $B_{n}(x) \sim n / 2$ and 2-normal if any blocks of $m$ digits appears with the frequency $2^{-m}$. Certain rational numbers such as $1 / 3=(0.01010101 \ldots)_{2}$ and certain artificial irrational numbers are simply normal in base 2 (or even 2-normal as in the case of the base 2 Champernowne number $\sum_{k \geq 1} k 2^{-c_{k}}$ with $c_{k}=k+$ $\sum_{j=1}^{k}\left\lfloor\log _{2} j\right\rfloor$ ), nobody knows whether this is the case for more classical numbers.

A simple method to provide lower bound of $B_{n}(x)$ for more 'natural' irrational numbers $x$ such as $e$ or $\pi$ is the following. Assume that $x$ has a finite irrationality exposant, that is, that there exists $\mu_{x} \in\left[2,+\infty\left[\right.\right.$ such that $|x-p / q| \geq q^{-\mu_{x}-\varepsilon}$ for any $\varepsilon>0$ and $q \gg_{\varepsilon} 1$. Then, we have that

$$
B_{n}(x) \geq \frac{\log (n)}{\log \left(\mu_{x}+\varepsilon\right)}+\mathcal{O}(1)
$$

for $n \gg_{\varepsilon} 0$. We can apply (1) to $e$ and $\pi$ because it has been proved that $\mu_{e}=2$ (Bundschuh [7] and Davis [8]) and $2 \leq \mu_{\pi} \leq 8.1$ (Hata [11]); surprisingly, these lower bounds for $B_{n}(e)$ and $B_{n}(\pi)$ are the best known to date. The proof of (1) runs as follows. Denoting by $p_{k} 2^{-n_{k}}$ the truncations of the binary expansion $\sum_{k=1}^{\infty} 2^{-n_{k}}$ of $x$, we have for all $k \gg_{\varepsilon} 0$

$$
\frac{1}{2^{\left(\mu_{x}+\varepsilon\right) n_{k}}} \leq\left|x-\frac{p_{k}}{2^{n_{k}}}\right| \leq \frac{1}{2^{n_{k+1}-1}},
$$

from which we deduce that $n_{k+1} \leq\left(\mu_{x}+\varepsilon\right) n_{k}+1$. Hence, $n_{k} \leq c_{\varepsilon, x}\left(\mu_{x}+\varepsilon\right)^{k}$ with $c_{\varepsilon, x}>0$. We now remark that

$$
\begin{aligned}
B_{n}(x) & =\#\left\{k: n_{k} \leq n\right\} \geq \#\left\{k: c_{\varepsilon, x}\left(\mu_{x}+\varepsilon\right)^{k} \leq n\right\} \\
& =\#\left\{k: k \leq\left(n-\log c_{\varepsilon, x}\right) / \log \left(\mu_{x}+\varepsilon\right)\right\}
\end{aligned}
$$

and (1) follows. See the final section for an elaboration of this method.

When $\alpha$ is a real irrational algebraic number, we can obtain the same kind of bound by using Roth's theorem [20] that $\mu_{\alpha}=2$. Ridout's theorem [18] (which claims that $\mu_{\alpha}=1$ when $q$ is restricted to a power of an integer) gives a better result than Roth's theorem but the improvement is marginal: for all $\varepsilon>0$, we have $B_{n}(\alpha) \geq \log (n) / \log (1+\varepsilon)+\mathcal{O}(1)$. However, a dramatic improvement was recently obtained by Bailey et al. [5], who proved that

$$
B_{n}(\alpha) \geq c_{\alpha} n^{1 / d}(1+o(1))
$$


for all real positive algebraic numbers $\alpha$ of degree $d \geq 2$ over $\mathbb{Q}$. Denoting by $a_{d} X^{d}+\cdots+a_{1} X+a_{0}$ the minimal polynomial of $\alpha$ over $\mathbb{Q}$, they obtained $c_{\alpha}=$ $\left(1+\log a_{d}\right)^{-1 / d}$ when $a_{0}<0$ and $a_{j} \geq 0$ otherwise (we call this 'case $\mathrm{S}$ ', for special, in the rest of the article). They only obtained $c_{\alpha}=\left(2 a_{d}\right)^{-1 / d}$ in the general case, whose proof is much more complicated (this result suggests an analogy with the Liouville-Thue-Siegel-Roth theorem concerning the diophantine approximation of algebraic numbers $\alpha$ of degree $d$ by rationals: (2) can be viewed as the analogue of Liouville's theorem, $\mu_{\alpha} \leq d$, and the expected bound that $B_{n}(\alpha) \gg n$ would be the analogue of Roth's theorem, $\mu_{\alpha}=2$ ): it seems that the use of Ridout's theorem instead of Roth's theorem in their proof improves the constant to $c_{\alpha}=a_{d}^{-1 / d}$. They used the bound (2) to prove that the number $\sum_{n \geq 1} 2^{-f(n)}$ is transcendental over $\mathbb{Q}$ provided that $f(n)$ grows faster than any power of $n$.

As a consequence of Theorem 1, we provide another proof of case S in Corollary 2 and also obtain a simpler proof of the bound $B_{n}(\alpha) \gg n^{1 / d}$ for a class of algebraic numbers $\alpha$ that do not fall obviously into case $\mathrm{S}$. We also note that a recent result of Adamczewski et al. [1, 2] implies in particular that the binary digits of algebraic real numbers cannot be generated by an automaton (see [3] for definitions). These two results are the most important towards the conjectural normality in all bases of algebraic numbers.

We now state some of the results proved in this article.

THEOREM 1. Let $x, y>0$ be real numbers, which are assumed irrational unless otherwise stated.

(i) If $x+y$ is irrational, we have for all $n \gg 0$,

$$
B_{n}(x+y) \leq B_{n}(x)+B_{n}(y)+1 .
$$

If $x+y \in \mathbb{N}$, then we replace $B_{n}(x+y)$ by $n+1$.

(ii) If $x y$ is irrational, we have for all $n \gg 0$,

$$
B_{n}(x y) \leq B_{n}(x) \cdot B_{n}(y)+\log _{2}\lfloor x+y+1\rfloor .
$$

If $y$ is an integer, we have $B_{n}(x y) \leq B_{n}(x) \cdot B(y)+B(y)$.

(iii) For all integers $A>0$, we have for all $n \gg 0$,

$$
B_{n}(x) \cdot B_{n}(A / x) \geq n-1-\left\lfloor\log _{2}(x+A / x+1)\right\rfloor .
$$

(iv) The three inequalities (3), (4) and (5) are sharp, up to a multiplicative factor for the last two.

REMARK. When $0<x, y<1$, results slightly better than (3) and (4) are indicated in [4, p. 157]: $B_{n}(x+y) \leq B_{n}(x)+B_{n}(y)$ and $B_{n}(x y) \leq B_{n}(x) \cdot B_{n}(y)$. On the other hand, inequality (iii) seems to be new. The results proved in Theorem 1 are sufficient for our purposes.

The sharpness of (3) (up to an additive constant) follows from the examples $x=\sum_{k \geq 1} 2^{-2^{k}}$ and $y=\sum_{k \geq 1} 2^{-3^{k}}$. We shall prove the sharpness of (4) and of (5) (which are less obvious) in Section 4 . Theorem 4 of that section answers a question in [5]. 
We obtain lower bounds of $B_{n}(\alpha)$ for certain classes of algebraic numbers $\alpha$ of the same form as (2) above.

COROLlary 2.

(i) Let $\alpha$ be an algebraic number in class $S$ of degree $d \geq 2$ and dominant coefficient $a_{d}$. We have that

$$
B_{n}(\alpha) \geq B\left(a_{d}\right)^{-1 / d} n^{1 / d}(1+o(1)) .
$$

(ii) Let $\beta$ be a positive irrational algebraic number, of degree $d \geq 2$, such that there exist two polynomials $P, Q$ with positive integers coefficients and two positive integers $a, b$ such that $P(\beta)=a+b Q(\beta)^{-1}$. Then,

$$
B_{n}(\beta) \geq(B(p) B(q))^{-1 / \delta} n^{1 / \delta}(1+o(1)),
$$

where $\delta=\operatorname{deg}(P Q)$ and $p, q$ are the dominant coefficients of $P$ and $Q$ respectively.

REMARKS. The lower bound in (i) is a small improvement on [5, Theorem 5.2] (proved with $1+\log \left(a_{d}\right)$ instead of our $B\left(a_{d}\right)$ ). It is presented here in order to illustrate the usefulness of the simple bounds in Theorem 1 .

The most favorable case in (ii) is when $P(X) Q(X)-a Q(X)-b$ is the minimal polynomial of $\beta$, in which case $\delta$ is the degree of $\beta$ over $\mathbb{Q}$. For example, the positive real root $\beta$ of the polynomial $8 x^{3}-2 x^{2}+4 x-3$, which is irreducible over $\mathbb{Q}$, satisfies the equation $4 \beta=1+2\left(2 \beta^{2}+1\right)^{-1}$ and therefore, by (ii), we have $B_{n}(\beta) \geq n^{1 / 3}(1+o(1))$. Note that $\beta$ does not belong to class $S$ and [5, Theorem 7.1] yields the weaker bound $B_{n}(\beta) \geq 16^{-1 / 3} n^{1 / 3}(1+o(1))$.

If we allow the case $b=0$ in (ii), we recover (i) provided that the constant term of $P$ is less than $a$. However, the proof of (ii) requires that $b>0$.

We now state a second application of Theorem 1. At first sight, it is not completely improbable that certain powers of a transcendental number $x$ might not be simply normal when $x$ itself is not simply normal in base 2: indeed, the rather unpredictable 'mixing' effect of carries might produce a smoothing 'law of large powers'. However, this is generally not the case as shown by the following result.

COROLlaRY 3. There exists a transcendental real number $\xi$ such that none of its powers $\xi^{j}\left(j \in \mathbb{N}^{\star}\right)$ is simply normal in base 2 .

REMARK. Let $\alpha$ be a real irrational algebraic number which is not of the form $\alpha=(a / b)^{1 / d}, a, b, d \in \mathbb{N}$. Then, for all integers $j \geq 1$, the number $\alpha^{j}$ is again a real irrational algebraic number and it is therefore expected that all of the powers of $\alpha$ are normal in all bases.

PROOF. It is enough to produce transcendental numbers $\xi$ such that, for example, $B_{n}(\xi) \ll \log (n)$ : inequality (4) implies that $B_{n}\left(\xi^{j}\right) \ll \log (n)^{j}$ for all integers $j \geq 1$, 
and thus the numbers $\xi^{j}$ all fail to be simply normal in base 2. Any one of the numbers $\xi=\sum_{n=1}^{\infty} a^{-b^{n}}$, with the integers $a \geq 2, b \geq 2$, is suitable. Its transcendence follows, for instance, from Roth's theorem when $b \geq 3$ and from Mahler's method when $b=2$ (see [17]).

Incidentally, Kempner [12] was the first to provide a transcendence proof of these numbers. When $b=2$, an unusual and original proof, based on the counting of nonzero digits of powers of $\xi^{j}$, was recently given by Knight [13]; as noticed by Bailey et al., the lower bound (2) provides a proof in the same 'digital' spirit.

\section{Warmup}

In this short section, we provide a proof of the subadditivity and submultiplicativity of the function $B$. We follow the presentation in [5].

2.1. Subadditivity of $\boldsymbol{B}$ Let $m$ denote a positive integer written in base 2 as $\left(m_{k} \ldots m_{0}\right)_{2}$ with $m_{k}=1$ and $m_{i} \in\{0,1\}$. Let $L_{j}$ denote the minimal integer $\ell \geq 0$ such that $m_{j+\ell}=0$. The usual rule of add with carry implies that

$$
B\left(m+2^{j}\right)=B(m)+1-L_{j} \leq B(m)+1 .
$$

If $n$ is a positive integer written as $n=\sum_{\ell=1}^{d} 2^{j \ell}$, then by successive applications of the previous equality, we obtain

$$
\begin{aligned}
B(m+n) & =B\left(m+\sum_{\ell=1}^{d} 2^{j_{\ell}}\right) \leq B\left(m+\sum_{\ell=1}^{d-1} 2^{j_{\ell}}\right)+1 \leq B\left(m+\sum_{\ell=1}^{d-2} 2^{j_{\ell}}\right)+2 \\
& \leq \cdots \leq B\left(m+\sum_{\ell=1}^{0} 2^{j_{\ell}}\right)+d=B(m)+d=B(m)+B(n) .
\end{aligned}
$$

2.2. Submultiplicativity of $\boldsymbol{B}$ We first remark that obviously $B\left(2^{j} m\right)=B(m)$. Therefore, with $n=\sum_{\ell=1}^{d} 2^{j_{\ell}}$,

$$
B(m n)=B\left(m \sum_{\ell=1}^{d} 2^{j_{\ell}}\right) \leq \sum_{\ell=1}^{d} B\left(2^{j_{\ell}} m\right)=\sum_{\ell=1}^{d} B(m)=B(m) B(n),
$$

where we have used the subadditivity of $B$.

\section{Proof of Theorem 1, parts (i), (ii) and (iii)}

For all integers $n \geq 0$, let $x_{n}=\left\lfloor 2^{n} x\right\rfloor, \quad y_{n}=\left\lfloor 2^{n} y\right\rfloor, \quad s_{n}=\left\lfloor 2^{n}(x+y)\right\rfloor$ and $p_{n}=\left\lfloor 2^{n} x y\right\rfloor$.

(i) For all $n \geq 0$, we have $x_{n}+y_{n} \leq s_{n}<x_{n}+y_{n}+2$, hence $s_{n}=x_{n}+y_{n}+z_{n}$ with $z_{n} \in\{0,1\}$. It follows that

$$
B\left(s_{n}\right)=B\left(x_{n}+y_{n}+z_{n}\right) \leq B\left(x_{n}\right)+B\left(y_{n}\right)+B\left(z_{n}\right) .
$$


Let us suppose that $x+y$ is irrational. Then, since

$$
B\left(s_{n}\right)=B_{n}(x+y), \quad B\left(x_{n}\right)=B_{n}(x), \quad B\left(y_{n}\right)=B_{n}(y) \quad \text { and } \quad B\left(z_{n}\right) \leq 1,
$$

we obtain

$$
B_{n}(x+y) \leq B_{n}(x)+B_{n}(y)+1 .
$$

If $x+y \in \mathbb{N}$, then $B_{n}(x)+B_{n}(y) \geq n$ because the sum of the $n$th digits after the point of $x$ and $y$ is 1 ( $x, y$ are irrational numbers).

(ii) We now show the second inequality.

We first assume that $x, y$ and $x y$ are irrational. Since there exist two real numbers $z_{n}$ and $\sigma_{n}$ satisfying

$$
x=\frac{x_{n}}{2^{n}}+\rho_{n}, \quad y=\frac{y_{n}}{2^{n}}+\sigma_{n} \quad \text { and } \quad 0<\rho_{n}, \quad \sigma_{n}<\frac{1}{2^{n}},
$$

we have

$$
0<x y-\frac{x_{n} y_{n}}{2^{2 n}}=\rho_{n} \frac{y_{n}}{2^{n}}+\sigma_{n} \frac{x_{n}}{2^{n}}+\rho_{n} \sigma_{n}<\frac{x+y+1 / 2^{n}}{2^{n}} .
$$

Furthermore, we have the binary expansion $x_{n} y_{n}=\sum_{\ell=0}^{2 n+n_{0}} \varepsilon_{\ell} 2^{\ell}$ where $n_{0} \in \mathbb{Z}$ (depending only on $x$ and $y$ ), $\varepsilon_{\ell} \in\{0,1\}$ and $\varepsilon_{2 n+n_{0}}=1$. For all $n \geq-n_{0}$, we write

$$
x_{n} y_{n}=\sum_{\ell=0}^{n-1} \varepsilon_{\ell} 2^{\ell}+\sum_{\ell=n}^{2 n+n_{0}} \varepsilon_{\ell} 2^{\ell}=a_{n}+2^{n} b_{n}
$$

with $0 \leq a_{n} \leq 2^{n}-1$. Thus, we obtain

$$
0<x y-\frac{b_{n}}{2^{n}}<\frac{x+y+1 / 2^{n}}{2^{n}}+\frac{a_{n}}{2^{2 n}} \leq \frac{x+y+1}{2^{n}} .
$$

We now remark that

$$
2^{n}\left(p_{n}+1\right)=2^{n}\left(\left\lfloor 2^{n} x y\right\rfloor+1\right)>\left\lfloor 2^{n} x\right\rfloor\left\lfloor 2^{n} y\right\rfloor=x_{n} y_{n} \geq 2^{n} b_{n},
$$

which implies that $p_{n}>b_{n}-1$ and, hence, $p_{n} \geq b_{n}$ (these are integers). Therefore, comparing the bound

$$
0<x y-\frac{p_{n}}{2^{n}}<\frac{1}{2^{n}}
$$

and the bound (6), we obtain that $p_{n}=b_{n}+v_{n}$ with $v_{n} \in\{0,1, \ldots,\lfloor x+y+1\rfloor\}$.

Finally, using the fact that $B\left(v_{n}\right) \leq \log _{2}\lfloor x+y+1\rfloor$ and that $B\left(x_{n} y_{n}\right) \geq B\left(b_{n}\right)$ (because $\left.B\left(x_{n} y_{n}\right)=B\left(a_{n}+2^{n} b_{n}\right)=B\left(a_{n}\right)+B\left(b_{n}\right) \geq B\left(b_{n}\right)\right)$, we obtain that

$$
\begin{aligned}
B\left(p_{n}\right)=B\left(b_{n}+v_{n}\right) & \leq B\left(b_{n}\right)+B\left(v_{n}\right) \\
& \leq B\left(x_{n} y_{n}\right)+\log _{2}\lfloor x+y+1\rfloor \\
& \leq B\left(x_{n}\right) \cdot B\left(y_{n}\right)+\log _{2}\lfloor x+y+1\rfloor,
\end{aligned}
$$


which is nothing but (4) because $B\left(p_{n}\right)=B_{n}(x y)$ (here we use the fact that $x y$ is irrational).

We now suppose that $x$ is irrational and that $y=2^{\ell_{1}}+\cdots+2^{\ell_{k}}$ is an integer. Then, by (i),

$$
\begin{aligned}
B_{n}(x y) & =B_{n}\left(2^{\ell_{1}} x+\cdots+2^{\ell_{k}} x\right) \leq B_{n}\left(2^{\ell_{1}} x\right)+\cdots+B_{n}\left(2^{\ell_{k}} x\right)+k \\
& =k \cdot B_{n}\left(2^{\ell_{1}} x\right)+k=B_{n}(x) \cdot B(y)+B(y) .
\end{aligned}
$$

This concludes the proof of (ii).

(iii) For all integer $n \geq 0$, letting $x_{n}=\left\lfloor 2^{n} x\right\rfloor$ and $y_{n}=\left\lfloor 2^{n} A / x\right\rfloor$, we have that

$$
0<u_{n}=x-\frac{x_{n}}{2^{n}}<\frac{1}{2^{n}} \quad \text { et } \quad 0<v_{n}=\frac{A}{x}-\frac{y_{n}}{2^{n}}<\frac{1}{2^{n}} .
$$

(The inequalities for $v_{n}$ hold because $A>0$.) Hence,

$$
A=x \cdot \frac{A}{x}=\left(\frac{x_{n}}{2^{n}}+u_{n}\right) \cdot\left(\frac{y_{n}}{2^{n}}+v_{n}\right)=\frac{x_{n} y_{n}}{2^{2 n}}+v_{n} \frac{x_{n}}{2^{n}}+u_{n} \frac{y_{n}}{2^{n}}+u_{n} v_{n}
$$

and

$$
0<v_{n} \frac{x_{n}}{2^{n}}+u_{n} \frac{y_{n}}{2^{n}}+u_{n} v_{n}<\frac{x+A / x+1}{2^{n}} .
$$

For $n \gg_{A, x} 1$, we have $1-(x+A / x+1) / 2^{n}>0$ and, thus,

$$
\begin{aligned}
\left\{\frac{x_{n} y_{n}}{2^{2 n}}\right\} & =\left\{A-v_{n} \frac{x_{n}}{2^{n}}-u_{n} \frac{y_{n}}{2^{n}}-u_{n} v_{n}\right\} \\
& =\left\{1-v_{n} \frac{x_{n}}{2^{n}}-u_{n} \frac{y_{n}}{2^{n}}-u_{n} v_{n}\right\}>1-\frac{x+A / x+1}{2^{n}} \\
& >1-\frac{1}{2^{n-\left\lfloor 1+\log _{2}(x+A / x+1)\right\rfloor}>0 .}
\end{aligned}
$$

(The fact that $A$ is an integer is used to invoke the 1-periodicity of the fractional part function $\{\cdot\}$.) These inequalities imply that we have at least

$$
B\left(x_{n} y_{n}\right) \geq n-1-\left\lfloor\log _{2}(x+A / x+1)\right\rfloor .
$$

Since $x$ and $A / x$ are irrational, we have $B\left(x_{n}\right)=B(x)$ and $B\left(y_{n}\right)=B(A / x)$. The submultiplicativity of $B$ finally yields that

$$
B_{n}(x) \cdot B_{n}(A / x) \geq n-1-\left\lfloor\log _{2}(x+A / x+1)\right\rfloor .
$$




\section{Proof of Theorem 1, part (iv)}

Borel proved that almost all real numbers are normal in all bases [16, p. 98] and thus the set of pairs of real numbers which are normal in base 2 is of full measure. Hence, for almost all pairs of irrational numbers $(x, y)$, inequality (4) is of the trivial form $2 n \leq n^{2}$ : therefore, we could expect to prove a stronger inequality. However, this is not the case; the following examples show that there exist pairs $(x, y)$ for which (4) is optimal (up to a multiplicative constant) without further hypothesis on $x$ and $y$. Let

$$
\mathscr{K}=\sum_{n=1}^{\infty} \frac{1}{2^{2^{n}}} \quad \text { and } \quad \mathscr{F}=\sum_{n=2}^{\infty} \frac{1}{2^{F_{n}}},
$$

where $\left(F_{n}\right)_{n \geq 0}$ is the Fibonacci sequence defined by $F_{1}=F_{2}=1$ and $F_{n+2}=F_{n+1}+$ $F_{n}$ for $n \geq 1$. Let $\phi=(1+\sqrt{5}) / 2$.

Theorem 4. As $n \rightarrow+\infty$,

$$
B_{n}(\mathscr{K}) \sim \frac{\log (n)}{\log (2)} \quad \text { and } \quad B_{n}\left(\mathscr{K}^{2}\right) \sim \frac{\log ^{2}(n)}{2 \log ^{2}(2)},
$$

as well as

$$
B_{n}(\mathscr{F}) \sim \frac{\log (n)}{\log (\phi)} \quad \text { and } \quad B_{n}\left(\mathscr{F}^{2}\right) \sim \frac{\log ^{2}(n)}{2 \log ^{2}(\phi)} .
$$

REMARKS. The powers of $\mathscr{K}$ are also considered in [14], where it is proved that their binary expansions are automatic.

The result for $\mathscr{F}^{2}$ answers the second question in [5, Section 'Open problems', p. 27]. More generally, numerical computations suggest that, for all $j \in \mathbb{N}^{\star}$,

$$
B_{n}\left(\mathscr{K}^{j}\right) \sim \frac{\log ^{j}(n)}{j ! \log ^{j}(2)} \quad \text { and } \quad B_{n}\left(\mathscr{F}^{j}\right) \sim \frac{\log ^{j}(n)}{j ! \log ^{j}(\phi)} .
$$

as $n \rightarrow+\infty$.

Proof of Theorem 4. The statements for $B_{n}(\mathscr{K})$ and $B_{n}(\mathscr{F})$ are clear. We now consider the cases of $\mathscr{K}^{2}$. We have

$$
\mathscr{K}^{2}=\left(\sum_{j=k \geq 1}+2 \sum_{j>k \geq 1}\right) \frac{1}{2^{2^{j}+2^{k}}}=\sum_{n=2}^{\infty} \frac{1}{2^{2^{n}}}+2 \sum_{m=1}^{\infty} \frac{e_{m}}{2^{m}},
$$

where $e_{m}$ is the number of ways of writing $m$ as $m=2^{j}+2^{k}$ with $j>k \geq 1$. Obviously, by uniqueness of the binary expansion, we have $e_{m} \in\{0,1\}$. Since the sets $P=\left\{2^{j}, j \geq 1\right\}$ and $Q=\left\{2^{j}+2^{k}-1, j>k \geq 1\right\}$ have empty intersection,

$$
\mathscr{K}^{2}=\sum_{n \in P \sqcup Q} \frac{1}{2^{n}} .
$$


Let $P \sqcup Q=R=\left\{R_{1}, R_{2}, \ldots\right\}$ : by definition, $B_{n}\left(\mathscr{K}^{2}\right)=\#\left\{k: R_{k} \leq n\right\}$. For all $\ell \geq 1$, there exist exactly $\ell-1$ elements in $Q \cap\left\{2^{\ell}+1, \ldots, 2^{\ell+1}\right\}$, which are of the form $2^{\ell}+2^{k}-1$ with $1 \leq k<\ell$. Hence, the set $Q \cap\left\{1,2, \ldots, 2^{n+1}\right\}$ has $\sum_{\ell=1}^{n}(\ell-1)=n(n-1) / 2$ elements. Furthermore, $P \cap\left\{1,2, \ldots, 2^{n+1}\right\}=n$, hence $R \cap\left\{1,2, \ldots, 2^{n+1}\right\}$ has $k_{n}=n(n+1) / 2$ elements, whose largest is $2^{n+1}$, that is, $R_{k_{n}}=2^{n+1}$.

Now let $j \geq 1$ be any integer: there exists an integer $n \geq 0$ such that $j \in\left\{k_{n}, k_{n}+\right.$ $\left.1, \ldots, k_{n+1}\right\}$. We then have

$$
\frac{2(n+1)}{(n+2)}=\frac{\log _{2}\left(R_{k_{n}}\right)^{2}}{k_{n+1}} \leq \frac{\log _{2}\left(R_{j}\right)^{2}}{j} \leq \frac{\log _{2}\left(R_{k_{n+1}}\right)^{2}}{k_{n}}=\frac{2(n+2)^{2}}{n(n+1)},
$$

which implies that $\lim _{j \rightarrow+\infty} \log \left(R_{j}\right)^{2} / j=2 \log ^{2}(2)$ and hence that

$$
\#\left\{k: R_{k} \leq n\right\}=\#\left\{k: \log ^{2}\left(R_{k}\right) \leq \log ^{2}(n)\right\} \sim \frac{\log ^{2}(n)}{2 \log ^{2}(2)},
$$

as desired.

For $\mathscr{F}^{2}$, we adapt the previous proof by using the fact that every positive integer $N$ can be written uniquely in the form $N=F_{n_{1}}+F_{n_{2}}+\cdots+F_{n_{k}}$, where the set $\left\{1<n_{1}<n_{2}<\cdots<n_{k}\right\}$ does not contain two consecutive integers. This expansion is known as Zeckendorf decomposition [22], Z-decomposition in short. We have

$$
\begin{aligned}
\mathscr{F}^{2} & =\left(\sum_{j=k \geq 2}+2 \sum_{\substack{j=k+1 \\
k \geq 2}}+2 \sum_{\substack{j \geq k+2 \\
k \geq 2}}\right) \frac{1}{2^{F_{j}+F_{k}}} \\
& =\sum_{n=2}^{\infty} \frac{1}{2^{F_{n}+F_{n}}}+\sum_{n=2}^{\infty} \frac{1}{2^{F_{n+1}+F_{n}-1}}+\sum_{\substack{j \geq k+2 \\
k \geq 2}} \frac{1}{2^{F_{j}+F_{k}-1}} \\
& =\sum_{n=2}^{4} \frac{1}{2^{2 F_{n}}}+\sum_{n=5}^{\infty} \frac{1}{2^{2 F_{n}}}+\sum_{n=4}^{\infty} \frac{1}{2^{F_{n}-1}}+\sum_{\substack{j \geq k+2 \\
k \geq 2}} \frac{1}{2^{F_{j}+F_{k}-1}} .
\end{aligned}
$$

We now show that the terms of the three infinite sums in (7) are pairwise distinct and thus that these three series provides the binary expansion of $\mathscr{F}^{2}$, up to the first finite sum, which implies a possible finite number of harmless carries.

For this, we note that the $Z$-decomposition of $2 F_{n}+1$ is $F_{n+1}+F_{n-2}+$ $F_{2}$ for $n \geq 5$ and that $F_{m}(m \geq 4)$ and $F_{j}+F_{k}(j \geq k+2, k \geq 2)$ are already $Z$-decompositions. We deduce that the equations

$$
\begin{gathered}
2 F_{n}=F_{m}-1(n \geq 5, m \geq 4), \quad 2 F_{n}=F_{j}+F_{k}-1(n \geq 5, j \geq k+2, k \geq 2) \quad \text { and } \\
F_{m}=F_{j}+F_{k}(m \geq 4, j \geq k+2, k \geq 2)
\end{gathered}
$$

have no solution, which proves the above claim. 
Thus, we have

$$
\mathscr{F}^{2}=\sum_{n=2}^{4} \frac{1}{2^{2 F_{n}}}+\sum_{m \in A_{1} \sqcup A_{2} \sqcup A_{3}} \frac{1}{2^{m}}
$$

where the sets

$$
\begin{gathered}
A_{1}=\left\{2 F_{n}, n \geq 5\right\}, \quad A_{2}=\left\{F_{m}-1, m \geq 4\right\} \quad \text { and } \\
A_{3}=\left\{F_{j}+F_{k}-1, j \geq k+2, k \geq 2\right\}
\end{gathered}
$$

have pairwise empty intersections. The rest of the proof is now similar to that of $\mathscr{K}^{2}$. We note that for $\ell \geq 4$, the set

$$
\begin{aligned}
A_{3} & \cap\left\{F_{\ell}+1, F_{\ell}+2, \ldots, F_{\ell+1}\right\} \\
& =\left\{F_{\ell}+F_{3}-1, F_{\ell}+F_{4}-1, \ldots, F_{\ell}+F_{\ell-2}-1\right\}
\end{aligned}
$$

has $\ell-4$ elements while

$$
A_{1} \cap\left\{F_{\ell}+1, F_{\ell}+2, \ldots, F_{\ell+1}\right\} \quad \text { and } \quad A_{2} \cap\left\{F_{\ell}+1, F_{\ell}+2, \ldots, F_{\ell+1}\right\}
$$

have (at most) one element. It follows that $B_{n}\left(\mathscr{F}^{2}\right) \sim \log ^{2}(n) / 2 \log ^{2}(\phi)$.

We now consider the optimality of (5). It will be enough to produce an irrational number $\mathscr{E}>0$ such that $B_{n}(\mathscr{E}) \asymp \sqrt{n}$ and $B_{n}(1 / \mathscr{E}) \asymp \sqrt{n}$ : such a number was mentioned in [5] (for another purpose) but no proof of the growth of $B_{n}(\mathscr{E})$ was provided. We therefore give one below. Starting from the binary expansion $n=$ $\left(x_{p} \ldots x_{1} x_{0}\right)_{2}$ of an integer $n$, we define the sets $S=\left\{n \in \mathbb{N}: \forall j \geq 0, x_{2 j}=0\right\}$ and $T=\left\{n \in \mathbb{N}: \forall j \geq 0, x_{2 j+1}=0\right\}$ (which satisfy $S \cap T=\{0\}$ and $2 T=S$ ) and then the real numbers

$$
\mathscr{E}=\prod_{n=0}^{\infty}\left(1+\frac{1}{2^{2^{2 n}}}\right)=\sum_{s \in S} \frac{1}{2^{s}} \quad \text { and } \quad \widehat{\mathscr{E}}=\prod_{n=0}^{\infty}\left(1+\frac{1}{2^{2^{2 n+1}}}\right)=\sum_{t \in T} \frac{1}{2^{t}} .
$$

Since any integer $n$ can be written exactly once under the form $n=s+t$ with $(s, t) \in S \times T$,

$$
\mathscr{E} \widehat{\mathscr{E}}=\sum_{(s, t) \in S \times T} \frac{1}{2^{s+t}}=\sum_{n=0}^{\infty} \frac{1}{2^{n}}=2
$$

The inverse of $\mathscr{E}$ is thus simply $\widehat{\mathscr{E}} / 2$ and it remains to check the property (which is probably well known, as it is given in [5] without proof) that $B_{n}(\mathscr{E}) \asymp \sqrt{n}$ and $B_{n}(\widehat{\mathscr{E}}) \asymp \sqrt{n}$. Only the upper bounds are useful for our purposes.

Proposition 5. As $n \rightarrow+\infty$, we have that

$$
\liminf _{n \rightarrow+\infty} \frac{B_{n}(\mathscr{E})}{\sqrt{n}} \geq 2 \sqrt{2 / 3}, \quad \limsup _{n \rightarrow+\infty} \frac{B_{n}(\mathscr{E})}{\sqrt{n}} \leq \sqrt{2}
$$


and

$$
\liminf _{n \rightarrow+\infty} \frac{B_{n}(\widehat{\mathscr{E}})}{\sqrt{n}} \geq 2 / \sqrt{3}, \quad \limsup _{n \rightarrow+\infty} \frac{B_{n}(\widehat{\mathscr{E}})}{\sqrt{n}} \leq 2
$$

In particular, for all $\varepsilon>0$ and all $n \gg_{\varepsilon} 0$, we have $B_{n}(\mathscr{E}) \cdot B_{n}(\widehat{\mathscr{E}} / 2) \leq(2 \sqrt{2}+\varepsilon) n$.

REMARK. These inequalities are probably not sharp.

PROOF OF PROPOSITION 5. Let us write the elements of the set

$$
S=\{0,2,8,10,32,34, \ldots\} \quad \text { as } \quad\left\{S_{0}<S_{1}<S_{2}<\ldots\right\}
$$

and those of

$$
T=\{0,1,4,5,16,17, \ldots\} \quad \text { as } \quad\left\{T_{0}<T_{1}<T_{2}<\ldots\right\}
$$

Clearly,

$$
\liminf _{n \rightarrow+\infty} \frac{B_{n}(\mathscr{E}, n)}{\sqrt{n}}=\limsup _{k \rightarrow+\infty} \frac{S_{k}}{k^{2}} \text { and } \quad \limsup _{n \rightarrow+\infty} \frac{B_{n}(\mathscr{E}, n)}{\sqrt{n}}=\liminf _{k \rightarrow+\infty} \frac{S_{k}}{k^{2}}
$$

and similar expressions for $\widehat{\mathscr{E}}$ and $T_{k}$. It can be easily proved that

$$
S_{k}=\sum_{j=0}^{\infty}\left(\left\lfloor\frac{k}{2^{j}}\right\rfloor \bmod 2\right) 2^{2 j+1}
$$

from which it follows that $S_{2^{n}}=2^{2 n+1}$ and $S_{2^{n}-1}=(2 / 3)\left(4^{n}-1\right)$. For all $k \in$ $\left\{2^{n}, \ldots, 2^{n+1}-1\right\}$, we thus have

$$
\frac{S_{2^{n}}}{\left(2^{n-1}-1\right)^{2}} \leq \frac{S_{k}}{k^{2}} \leq \frac{S_{2^{n+1}-1}}{2^{2 n}}
$$

Hence,

$$
\liminf _{k \rightarrow+\infty} \frac{S_{k}}{k^{2}} \geq \lim _{n \rightarrow+\infty} \frac{S_{2^{n}}}{\left(2^{n+1}-1\right)^{2}}=\frac{1}{2} \quad \text { and } \quad \limsup _{k \rightarrow+\infty} \frac{S_{k}}{k^{2}} \leq \lim _{n \rightarrow+\infty} \frac{S_{2^{n+1}-1}}{\left(2^{n}\right)^{2}}=\frac{8}{3} .
$$

Since $T=S / 2$, we also deduce that

$$
\liminf _{k \rightarrow+\infty} \frac{T_{k}}{k^{2}} \geq \frac{1}{4} \quad \text { and } \quad \limsup _{k \rightarrow+\infty} \frac{T_{k}}{k^{2}} \leq \frac{4}{3} .
$$

This completes the proof. 
The transcendence of $\mathscr{E}$ and $\widehat{\mathscr{E}}$ can be proved thanks to Mahler's method: the proof can be found in an article of Blanchard and Mendès-France [6]. It is, in fact, easy to produce other pairs of numbers which are the inverse of each other and whose binary expansions are explicitly known: given any given subset $A$ of $\mathbb{N}$, we can use the identity

$$
\prod_{n \in A}\left(1+z^{2^{n}}\right) \times \prod_{n \in \mathbb{N} \backslash A}\left(1+z^{2^{n}}\right)=\prod_{n \in \mathbb{N}}\left(1+z^{2^{n}}\right)=\frac{1}{1-z} .
$$

This suggests looking at the inverse of real numbers with few 1's in base 2: if $\psi: \mathbb{N} \rightarrow \mathbb{N}$ is strictly increasing, of inverse $\psi^{[-1]}$, let $\mathscr{D}_{\psi}=\sum_{n=0}^{\infty} 2^{-\psi(n)}$ :

$$
B_{n}\left(1 / \mathscr{D}_{\psi}\right) \geq \frac{n}{\psi^{[-1]}(n)}(1+o(1)) .
$$

A related problem, due to Mendès-France, is to prove (or disprove) the existence of an irrational number simply normal in base $b$ whose inverse is not simply normal or numbers with this property with respect to normality in base $b$ or absolute normality. Clearly, the answer is positive in the case of simple normality in base 2 if we

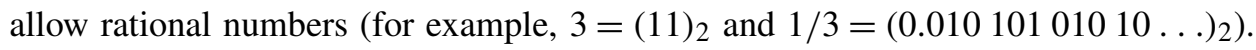
It would be equally interesting to prove (or disprove) the existence a real number $x$ such that both $x$ and $1 / x$ are simply normal in base $b$ (or $b$-normal or normal in all bases).

\section{Proof of Corollary 2}

(i) Since $\alpha$ is of degree $d \geq 2$, the number $\left|a_{0}\right| / \alpha$ is irrational, as are the numbers $a_{j} \alpha^{j-1}$ for those $j \in\{2, \ldots, d\}$ such that $a_{j} \neq 0$. Since

$$
\left|a_{0}\right| \alpha^{-1}=a_{1}+a_{2} \alpha+\cdots+a_{d} \alpha^{d-1},
$$

the various inequalities in Theorem 1 imply that

$$
\begin{aligned}
B_{n}\left(\left|a_{0}\right| \alpha^{-1}\right) & \leq d+B\left(a_{1}\right)+B_{n}\left(a_{2} \alpha\right)+\cdots+B_{n}\left(a_{d} \alpha^{d-1}\right) \\
& \leq d+B\left(a_{1}\right)+B\left(a_{2}\right)\left(B_{n}(\alpha)+1\right)+\cdots+B\left(a_{d}\right)\left(B_{n}\left(\alpha^{d-1}\right)+1\right) \\
& \leq B\left(a_{d}\right) B_{n}(\alpha)^{d-1}(1+o(1)) .
\end{aligned}
$$

The last inequality holds because $B_{n}(\alpha) \rightarrow+\infty$ as $n \rightarrow+\infty$. Furthermore, we have that

$$
B_{n}\left(\left|a_{0}\right| \alpha^{-1}\right) \geq \frac{n}{B_{n}(\alpha)}-c_{0}
$$

with $c_{0}>0$ independent of $n$. Therefore

$$
B\left(a_{d}\right) B_{n}(\alpha)^{d}(1+o(1))+c_{0} B_{n}(\alpha) \geq n
$$


and we finally obtain that

$$
B_{n}(\alpha) \geq B\left(a_{d}\right)^{-1 / d} n^{1 / d}(1+o(1))
$$

as desired.

(ii) Since $a$ and $b$ are positive integers,

$$
B_{n}(P(\beta))=B_{n}(a+b / Q(\beta)) \geq B_{n}(b / Q(\beta))-c_{1} \geq \frac{n}{B_{n}(Q(\beta))}-c_{2}
$$

where $c_{1}>0, c_{2}>0$ do not depend on $n$. (In the right most inequality, we use the fact that $b>0$.) Hence,

$$
\left(B_{n}(P(\beta))+c_{2}\right) B_{n}(Q(\beta)) \geq n
$$

and essentially the same argument as above yields that

$$
B(p) B_{n}(\beta)^{\operatorname{deg}(P)} B(q) B_{n}(\beta)^{\operatorname{deg}(Q)} \geq n(1+o(1)) .
$$

The assertion follows.

\section{Binary expansion of roots of analytical functions}

One might wonder how to extend the lower bounds in Corollary 2 to the case of roots of analytical functions. This could provide nontrivial information for numbers such as $1-e$ and $\pi$ which are solutions of the equations $\log (1-z)=1$ and $\sin (z)=$ 0 . Such results exist but are far from satisfactory; therefore, we quote them without details.

Let $F$ be a power series of radius of convergence $R>0$,

$$
F(z)=\sum_{n=1}^{\infty} a_{n} z^{b_{n}},
$$

with $a_{n}=p_{n} / q_{n} \in \mathbb{Q}^{+}$and $\left(b_{n}\right)_{n \geq 1}$ a strictly increasing sequence of integer. Let $d_{n}=\operatorname{lcm}\left(q_{0}, q_{1}, \ldots, q_{n}\right), \omega(n)=\log _{2}\left(d_{n}\right)$ and

$$
\left|\sum_{n=k+1}^{\infty} a_{n} z^{b_{n}}\right| \leq 2^{-\Omega_{z}(k)},
$$

where the function $k \mapsto \Omega_{z}(k)$ is assumed to be strictly increasing, with inverse $k \mapsto \Omega_{z}^{[-1]}(k)$.

THEOREM 6. For any rational $\rho>0$, let $\xi_{\rho}$ denote the unique real number $\xi \in[0, R[$ such that $F(\xi)=\rho$. Then, with $k=\Omega_{\xi_{\rho}}^{[-1]}(n)$,

$$
B_{n}\left(\xi_{\rho}\right) \geq B\left(d_{k} a_{k}\right)^{-1 / k} \cdot(n-\omega(k))^{1 / k}(1+o(n)) .
$$

The proof is a straightforward adaptation of that of [5, Theorem 5.2]. Nothing interesting occurs if $b_{n}$ grows slower than $c^{n}$. With $a_{n}=1$ and $b_{n}=2^{n^{2}}$, we have $B_{n}\left(\xi_{\rho}\right) \gg \exp \left(c_{\rho} \sqrt{\log (n)}\right)$. When $a_{n}=1$, the faster $b_{n}$ grows, the closer $B_{n}\left(\xi_{\rho}\right)$ is to $n$. 


\section{Further links between diophantine approximation and normality}

We conclude this article with some reflections that could lead, in certain cases, to refinements of (1):

$$
B_{n}(x) \geq \frac{\log (n)}{\log \left(\mu_{x}+\varepsilon\right)}+\mathcal{O}(1)
$$

for $n \gg_{\varepsilon} 0$ for irrational numbers $x$ with finite irrational exposant $\mu_{x}$ (that is, nonLiouville numbers). We remark that the proof of (1) only partially uses the strength of the irrationality measure $|x-p / q| \geq q^{-\mu_{x}-\varepsilon}$ (valid for all rational $p / q$ with $q \gg_{\varepsilon} 1$ ). Indeed, we used this measure only when $q$ is a power of 2 . Therefore, it is enough to use an irrationality measure of the following form: given an irrational number $x$ and an integer $b \geq 2$, there exists $v_{x, b} \geq 1$ such that for all $m \gg_{\varepsilon} 0$

$$
\left|x-\frac{p}{b^{m}}\right| \geq \frac{1}{b^{\left(v_{x, b}+\varepsilon\right) m}} .
$$

We deduce a small improvement of (1):

$$
B_{n}(x) \geq \frac{\log (n)}{\log \left(v_{x, 2}+\varepsilon\right)}+\mathcal{O}(1) .
$$

For example, Ridout's theorem yields $v_{\alpha, b}=1$ for all irrational algebraic numbers $\alpha$ and all $b \geq 2$, where Roth's theorem only yields $v_{\alpha, b} \leq 2$. The situation is worse for numbers such as $e$ or $\pi$ because, for all $b \geq 2$, we only know the trivial upper bounds $v_{e, b} \leq \mu_{e}=2$ and $v_{\pi, b} \leq \mu_{\pi} \leq 8.1$ : we cannot improve on the lower bounds for $B_{n}(e)$ and $B_{n}(\pi)$ provided by (1) when $b=2$. On a more positive note, the author recently proved in [19] that for the numbers $x=\log (1-1 / b)$, we have $v_{x,|b|} \rightarrow 1$ as $b \rightarrow \pm \infty$ but this is clearly not as good as Ridout's theorem for algebraic numbers.

In the most favorable case where $v_{x, 2}=1$, the presence of $\varepsilon$ rules out the possibility of getting something better than $B_{n}(x) \gg \log (n)$. However, as we now show, an improvement would occur if, in (8), we could replace $q^{1+\varepsilon}$ by a more explicit function of $q$. It is not difficult to prove that, given a positive-valued and nonincreasing function $\psi$ defined on the powers $\left(b^{m}\right)_{m \geq 0}$, for almost all $x$, the equation

$$
\left|x-\frac{p}{b^{m}}\right|<\frac{\psi\left(b^{m}\right)}{b^{m}}
$$

admits finitely many solutions $(p, m) \in \mathbb{Z} \times \mathbb{N}$ if the series $\sum_{m \geq 0} \psi\left(b^{m}\right)$ converges (this result is a particular case of more general results: see [10, Ch. 2]). The proof runs as follows: we restrict $x \in[0,1[$ and consider the set

$$
E_{m}=\left[0,1\left[\bigcap \bigcup_{p=0}^{b^{m}-1}\right] \frac{p-\psi\left(b^{m}\right)}{b^{m}}, \frac{p+\psi\left(b^{m}\right)}{b^{m}}[.\right.
$$


The set $E$ of those $x \in[0,1$ [ such that (9) admits infinitely many solutions $(p, m)$ is $E=\bigcap_{n \geq 0} \bigcup_{m \geq n} E_{m}$ and to conclude it is enough to prove that the Lebesgue measure $\lambda(E)$ of $\bar{E}$ is 0 . However,

$$
\lambda(E) \leq \sum_{m=n}^{\infty} \lambda\left(E_{m}\right) \leq 2 \sum_{m=n}^{\infty} \psi\left(b^{m}\right) \rightarrow 0
$$

as $n \rightarrow+\infty$ by hypothesis.

The converse is also true but more difficult to prove: this a consequence of a theorem of Duffin-Schaeffer [9, p. 245], see also [10, Theorem 2.9]. Hence, with $\psi(q)=1 /(\log (q) \log \log (q))$, (9) admits infinitely many solutions and, as pointed out by Harman, this implies that, for almost all $x$, there exist infinitely many $n$ such that $B_{n+m}(x)=B_{n}(x)+m$ for $m=\left\lfloor\lambda \log _{2}(n)\right\rfloor$ when $\lambda=1$ and only finitely many $n$ when $\lambda>1$.

On the other hand, for all $\rho>1$, (9) admits almost surely only finitely many solutions with $\psi(q)=1 / \log (q)^{\rho}$ and we can also draw some conclusions on $B_{n}(x)$ from this when $b=2$.

PROPOSITION 7. Let $x$ be an irrational number. We suppose that there exists a constant $\rho>1$ (depending at most on $x$ ) such that, for all $p \in \mathbb{Z}$ and $m \in \mathbb{N}$ with $m \gg 0$,

$$
\left|x-\frac{p}{2^{m}}\right| \geq \frac{1}{2^{m} \log ^{\rho}\left(2^{m}\right)} .
$$

Then,

$$
B_{n}(x) \geq \frac{n}{\rho \log (n)}(1+o(1)) .
$$

REMARK. Since for almost all $x$, estimate (10) holds, we also have that, for almost all $x$, (11) holds. However, we already know that, for almost all $x$, the much stronger estimate $B_{n}(x) \sim n / 2$ holds. Furthermore, it seems difficult to deduce something better than (11) from (10) without further information on $x$. Thus, general methods based on diophantine inequalities such as (10) are probably not strong enough to prove the normality of natural constants. On the other hand, estimates of the form (10) might be simpler to prove than normality.

PROOF. We denote by $p_{k} 2^{-n_{k}}$ the truncations of the binary expansion $\sum_{k=1}^{\infty} 2^{-n_{k}}$ of $x$. For all $k \gg 0$,

$$
\frac{1}{2^{n_{k}} \log ^{\rho}\left(2^{n_{k}}\right)} \leq\left|x-\frac{p_{k}}{2^{n_{k}}}\right| \leq \frac{1}{2^{n_{k+1}-1}},
$$

which implies that

$$
n_{k+1} \leq n_{k}+\rho \log \left(n_{k}\right)+\rho \log \log (2)+1 \quad \text { for } k \geq k_{0} .
$$

Clearly, we have $n_{k} \leq u_{k}$ where $u_{k}$ is defined by

$$
u_{k_{0}}=n_{k_{0}} \quad \text { and } \quad u_{k+1}=u_{k}+\rho \log \left(u_{k}\right)+\rho \log \log (2)+1
$$


and it will be sufficient to bound $u_{k}$. We remark that in the absence of further information on $x$, bounding $u_{k}$ is in fact the best we can do here since we cannot exclude that $n_{k+1} \geq n_{k}+\rho \log \left(n_{k}\right)+\mathcal{O}(1)$.

Below we prove the following fact. For any $a \geq 1$ and $b>0$, let $v_{k}$ be defined by $v_{1}=a$ and $v_{k+1}=v_{k}+\log \left(v_{k}\right)+b$ (the conditions on $a$ and $b$ ensure that $v_{k}$ is defined for all $k \geq 1)$. Then there exists a constant $d=d(a, b)$ such that

$$
v_{k} \leq k \log (k+d)+k \log \log (k+d)+b k .
$$

Applying (12) to $v_{n}=u_{n+k_{0}} / \rho$ (with a suitable $b$ ), we obtain that $u_{k} \leq \rho k \log (k)+$ $g(k)$ with $g(k)=\mathcal{O}(k \log \log (k))$ for $k \geq 1$. Hence,

$$
\begin{aligned}
B_{n}(x) & =\#\left\{k \geq 1: n_{k} \leq n\right\} \\
& \geq \#\left\{k \geq 1: u_{k} \leq n\right\} \\
& \geq \#\{k \geq 1: \rho k \log (k)+g(k) \leq n\} \sim R(n)
\end{aligned}
$$

where $R$ is the inverse function of $k \mapsto \rho k \log (k)+g(k)$. Since $R(k) \sim k /(\rho \log (k))$ as $k \rightarrow+\infty$, Equation (11) follows.

It remains to prove (12). We choose $d$ such that firstly

$$
\log (d+1)+b+\log \log (d+1) \geq a
$$

and, secondly, for all $k \geq 1$,

$$
b+\log \log (d+k) \leq \log (d+k)
$$

(obviously, this is possible if $d$ is large enough with respect to $b$ ). The inequality $\log (1+x) \leq x$ for all $x>-1$ and (14) imply that, for all $k \geq 1$,

$$
\log \left(1+\frac{b+\log \log (k+d)}{\log (k+d)}\right) \leq 1 .
$$

By calculus, one proves that

$$
k \log (k+d)+\log (k)+1 \leq(k+1) \log (k+d+1) .
$$

Inequalities (15) and (16) will be implicitly used below. We now proceed by induction. We first remark that (13) implies that (12) is true for $k=1$. We suppose that (12) is true for $k$. Then,

$$
\begin{aligned}
v_{k+1} \leq & k \log (k+d)+k \log \log (k+d)+b k \\
& +\log (k \log (k+d))+\log \left(1+\frac{b+\log \log (k+d)}{\log (k+d)}\right)+b \\
\leq & k \log (k+d)+\log (k)+1+(k+1) \log \log (k+d)+b(k+1) \\
\leq & (k+1) \log (k+d+1)+(k+1) \log \log (k+d+1)+b(k+1),
\end{aligned}
$$

which completes the proof of the induction. 


\section{Acknowledgements}

I warmly thank M. Waldschmidt for his critical reading of the first version of this article and G. Harman for answering some of my questions and suggesting certain references.

\section{References}

[1] B. Adamczewski and Y. Bugeaud, 'On the complexity of algebraic numbers I. Expansions in integer bases', Ann. Math. 165(2) (2007), 547-565.

[2] B. Adamczewski, Y. Bugeaud and F. Luca, 'Sur la complexité des nombres algébriques', C. R. Acad. Sci. Paris 339 (2004), 11-14.

[3] J.-P. Allouche and J. Shallit, Automatic Sequences. Theory, Applications, Generalizations (Cambridge University Press, Cambridge, 2003).

[4] D. H. Bailey and J. M. Borwein, Mathematics by Experiments: Plausible Reasoning in the 21st Century (A. K. Peters, Natick, MA, 2004).

[5] D. H. Bailey, J. M. Borwein, R. E. Crandall and C. Pomerance, 'On the binary expansions of algebraic numbers', J. Théor. Nombres Bordeaux 16(3) (2004), 487-518.

[6] A. Blanchard and M. Mendès-France, 'Symétrie et transcendance', Bull. Sci. Math. (2) 106(3) (1982), 325-335.

[7] P. Bundschuh, 'Irrationalitätsmaße für $e^{a}, a \neq 0$ rational oder Liouville-Zahl', Math. Ann. 192 (1971), 229-242.

[8] C. S. Davis, 'Rational approximations to e', J. Aust. Math. Soc. 25 (1978), 497-502.

[9] R. J. Duffin and A. C. Schaeffer, 'Khintchine's problem in metric diophantine approximation', Duke Math. J. 41 (1941), 241-255.

[10] G. Harman, Metric Number Theory, London Mathematical Society Monographs, 18 (Clarendon Press/Oxford University Press, New York, 1998).

[11] M. Hata, 'Rational approximations to $\pi$ and some other numbers', Acta Arith. 63(3) (1993), $335-349$.

[12] A. J. Kempner, 'On transcendental numbers', Trans. Amer. Math. Soc. 17 (1916), 476-482.

[13] M. J. Knight, 'An 'ocean of zeroes' proof that a certain non-Liouville number is transcendental', Amer. Math. Monthly 98 (1991), 947-949.

[14] S. Lehr, J. Shallit and J. Tromp, 'On the vector space of the automatic reals', Theoret. Comput. Sci. 163(1-2) (1996), 193-210.

[15] B. Lindström, 'On the binary digits of a power', J. Number Theory 65(2) (1997), 321-324.

[16] I. Niven, Irrational Numbers, The Carus Mathematical Monographs, 11 (1967).

[17] K. Nishioka, Mahler Functions and Transcendence, Lecture Notes in Mathematics, 1631 (Springer, Berlin, 1996).

[18] D. Ridout, 'Rational approximations to algebraic numbers', Mathematika 4 (1957), 125-131.

[19] T. Rivoal, 'Convergents and irrationality measures of logarithms', Rev. Mat. Iberoamericana 23(3) (2007), 931-952.

[20] K. F. Roth, 'Rational approximations to algebraic numbers', Mathematika 2 (1955), 1-20 (Corrigendum 168)

[21] K. B. Stolarsky, 'The binary digits of a power', Proc. Amer. Math. Soc. 71(1) (1978), 1-5.

[22] E. Zeckendorf, 'Représentation des nombres naturels par une somme des nombres de Fibonacci ou de nombres de Lucas', Bull. Soc. Roy. Sci. Liège 41 (1972), 179-182.

TANGUY RIVOAL, Institut Fourier, CNRS UMR 5582/Université Grenoble 1, 100 rue des Maths, BP 74, 38402 Saint-Martin d'Hères cedex, France e-mail: rivoal@ujf-grenoble.fr 